\title{
Realization of Circular Slot Frequency Selective Surfaces using Photoplotter and Wet Etching Technique for Terahertz Material Sensing Applications
}

\author{
N.S.Ishak ${ }^{1}$, M. Hashim Dahri' ${ }^{1}$, N.A.Awang ${ }^{2}$, W.Y.Chong ${ }^{3}$, F.C.Seman ${ }^{\text {* }}$ \\ ${ }^{1}$ Faculty of Electrical and Electronic Engineering, \\ Universiti Tun Hussein Onn Malaysia, 86400 Parit Raja, Batu Pahat, Johor, MALAYSIA \\ ${ }^{2}$ Faculty of Applied Science and Technology (FAST), \\ Universiti Tun Hussein Onn Malaysia, 86400 Parit Raja, Batu Pahat, Johor, MALAYSIA \\ ${ }^{3}$ Photonics Research Centre, Deputy Vice Chancellor (Research \& Innovation) Office, \\ University of Malaya, 50603 Kuala Lumpur, MALAYSIA \\ *Corresponding Author
}

DOI: https://doi.org/10.30880/ijie.2020.12.06.006

Received 04 September 2020; Accepted 29 May 2020; Available online 02 July 2020

\begin{abstract}
This paper discusses on the analysis of band pass Frequency Selective Surfaces (FSS) for performance enhancement in material sensing application. Terahertz Spectroscopy has proved to be versatile tool for detection and sensing in measuring non-conductive materials. It is because most of the non-conductive materials have unique molecular resonance that may translate as transmission and absorption of signals within terahertz range. However, the most critical issue in detection and sensing is to improve its sensitivity therefore an extremely low concentration material still can be able to be detected in $\mathrm{THz}$ band. Hence, in this paper, a circular slot is modeled on a planar structure of Rogers Duroid 5880LZ substrate with thickness of 508 $\mu$ m using Computer Simulation Technology (CST). The simulation generates a band pass response with transmission magnitude of 0.95 at $0.66 \mathrm{THz}$. Furthermore, photoplotter and wet etching fabrication process is used for the realization of terahertz FSS. Simulated and measured transmission shows a good agreement between $0.5 \mathrm{THz}$ to $0.7 \mathrm{THz}$ as only $1 \%$ shifts in frequency between simulated and measured results. Besides that, the fabrication of circular FSS shows narrower measured bandwidth as compared to its simulated counterpart. Hence, with the limitation of the wet etching to produce micron size structure both simulation and measured result shows good agreement for all the critical issues in this study.
\end{abstract}

Keywords: Terahertz, Frequency Selective Surfaces

\section{Introduction}

Terahertz radiation is in spectral region of $0.1 \mathrm{THz}$ to $10 \mathrm{THz}$ between microwave and infrared part of the electromagnetic spectrum. Terahertz radiation can propagate through wide range of dielectric materials [1]. This sparked many researchers' interest to further explore the potentiality of characterization, detection and sensing studies at terahertz range. Recently, Terahertz Time-Domain Spectroscopy has emerged as an attractive technique that enables the label-free detection on chemical and biological compounds [2]. It is because, the most of non-conductive materials such as chemical and biological compounds have unique molecular resonance that able to translate as transmission or absorption peak at terahertz range. Past studies show that, terahertz spectroscopy is more suitable in sensing, detection and characterization of various forms of material state, such as illicit drugs and explosive substances, alcohol and antibiotics contents, fructose 
and glucose solution [2]-[5]. However, the main issue that prevents the widespread use of THz-TDS in material sensing and detection application, despite the contributions of Terahertz Spectroscopy is limited sensitivity in material detection and sensing application. With this limitation in material sensitivity, several researchers have proposed that, by adding metamaterial layers the sensitivity can be achieved. Studies shows that, the sensitivity can be improved by a factor of $10^{4}$ to $10^{5}$ when a metamaterial layers has been added [6]-[8]

Metamaterial structure is also known as Frequency Selective Surface (FSS), which consists of periodic array of slots or patch elements on a dielectric substrate with no ground plane. The phase response of FSS depends on the size adjustment of the elements of FSS. FSSs are widely used in EM shielding, reflectors, absorbers and recently been used as transducers in biochemical sensing, which allow the detection of various materials with increasing its sensitivity [2] [9]. There are several geometries in FSS such as, dipole, hexagon ring and circular structure. According to Ben A Munk [10], FSS can be categorized into four groups such as, center connected, loop types, solid interiors and combinations. Each group and structures of FSS produces their own characteristics response according to their applications. Besides that, because of their unlimited FSS structures, there is no specific method to realize FSS. Therefore, FSS can be precisely machined by laser technology, direct writing technique, or using microfabrication process method [11]-[13]. A few previous studies have described their work on fabrication techniques by using laser technology, photothermal direct writing, and optical lithography with chemical etching in different materials. Each fabrication technique shows closely resemblance in terms of transmittance magnitude of $0.90,0.63$, and 0.40 respectively. Previous studies show that, the fabrication using chemical (wet) etching has the lowest transmittance magnitude among other fabrication techniques mentioned, and thus it is the least preferred technique to be applied in terahertz region fabrication [10]-[12]. Meanwhile, it is quite challenging to produce a pattern working at terahertz region as the dimension can be smallest as $10 \mu \mathrm{m}$ and there are not so many papers that had fabricated FSS using wet etching technique because of the limitation in its measurement technique itself.

Therefore, in this paper, the realization of the circular slot FSS is achieved by photoplotter and wet etching techniques. It is well known that, it has limitation to produce micron size structures, but the realization can be performed due to its proper observation and systematic fabrication setup. This technique offers simple and basic structure to be applied using wet etching technique and has shown good agreement between measured and simulated result with transmittance magnitude and peak of 0.871 and $87 \%$ between $0.5 \mathrm{THz}$ to $0.7 \mathrm{THz}$ frequencies. Furthermore, throughout this study the emphases have been given to FSS slot with band pass characteristics, because it is more suitable in material sensing applications.

\section{FSS-Based Filter Design}

Among different kinds of FSSs which can form the resonant unit of terahertz filter, basic and simple structure of circular geometry is chosen. Because of its symmetric structure, the frequency response characteristics do not affect even with different propagation modes of TE and TM. Furthermore, due to its symmetrical structure, total distance of one loop is equal to one wavelength which is represented as the circumference of circle. Thus, resonant frequency of a circular slot FSS can be expressed as in equation 1 [14].

$$
f_{r}=\frac{c}{\lambda \sqrt{\varepsilon_{r}}}=\frac{c}{\pi d \sqrt{\varepsilon_{r}}}
$$

Moreover, FSS could increase the signal concentration of $\mathrm{THz}$ and at the same time, it may able to increase the detection abilities in material sensing [2], [15], [16]. This is demonstrated by observing the surface current intensity with and without FSS as shown in Fig. 1. It is clearly seen from Fig. 1 (a), which is represented by a vacuum model in the 3D simulation, that, the vacuum medium acts as an exposed material at the free-space medium. Surface current intensity without FSS shows weak intensity as compared to the surface current intensity shown in Fig. 1 (b) when FSS structure is applied onto the vacuum medium through simulation. Thus, it can show the importance of surface current intensity with and without FSS structure for material sensing purposes as by applying the FSS structures, it may help to enhance the detection sensitivity of material sensing. It is observed through the simulations that, by adding circular slot into FSS the surface current is increased by $40.8 \%$. Thus, this demonstration shows that, the FSS able to increase the detection abilities in material sensing. 
a

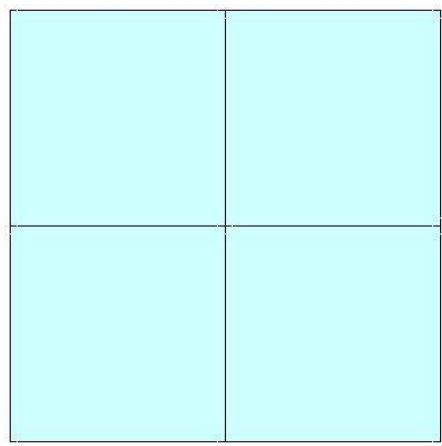

b

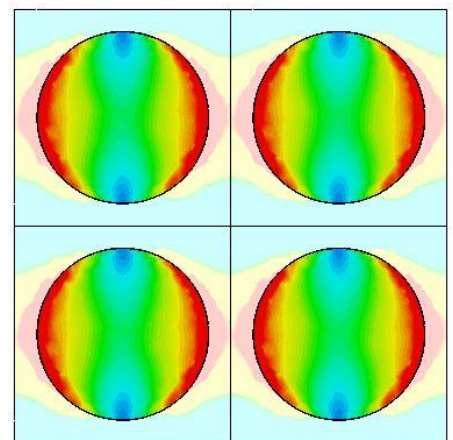

Fig. 1 - (a) Surface current intensity without FSS layer (b) Surface current intensity with FSS layer.

In this work, the unit cell geometry of circular slot FSS is designed and simulated by CST Microwave Studio at a frequency range of $0.5 \mathrm{THz}$ to $0.7 \mathrm{THz}$ and the pass band at $-3 \mathrm{~dB}(0.707$ magnitude) from frequency with resonant frequency of $0.66 \mathrm{THz}$ and with a magnitude of transmittance of 0.950 . The simulation with commercially available Microwave-Computer Simulation Technology (MW-CST) had been setup with unit cell boundary conditions and Frequency Domain Solver, which is more suitable to optimize high resonance and electrically small structure such FSS. The circular slot FSS is designed with planar structure of Roger as substrate layer. Roger Duroid RT5880 LZ is chosen as the substrate to support the FSS structure because of its stable dielectric constant of $\varepsilon_{\mathrm{r}}=2$ and low loss tangent of 0.0021 , with a thickness $T_{s}$ of $508 \mu \mathrm{m}$. The conducting layer of circular slot FSS is made up with copper thickness of $35 \mu \mathrm{m}$.

The periodicity of the FSS is denoted by $D y=D x$ and the diameter size of circular slot is taken as $d$. All-important parameters involved in the design can be seen in Fig. 2.
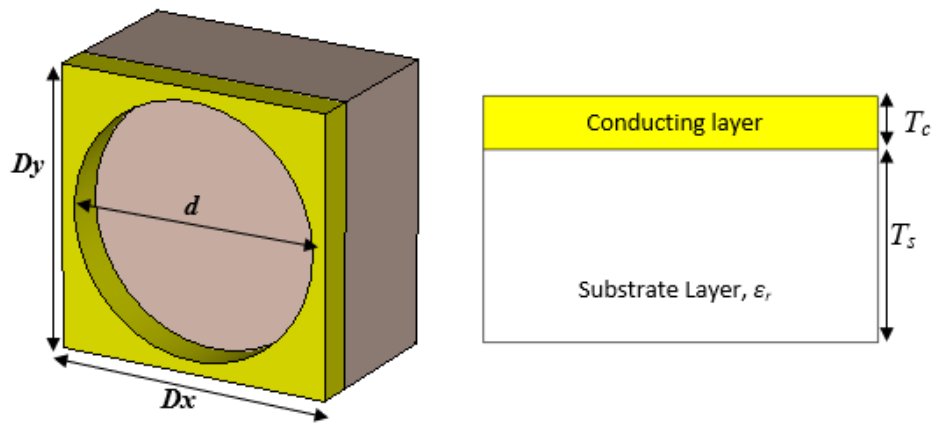

Fig. 2 - Unit cell of FSS and side view of FSS.

\section{Fabrication Process}

Photoplotter and wet etching is used for realization of investigated circular FSS. Therefore, the process of preparing masking film has been done using Photoplotter (FilmStar-PLUS). Photoplotter is used to pattern the circular structure FSS in order to have minimal error and maintain uniform pattern. It uses laser-diode (red light, $670 \mathrm{~nm}$ wavelength) light from photoplotter to print the geometrical design onto the photoresist layer. The used photoresist films are highly sensitive to the sunlight. Thus, this process is performed in a dark room. As for PCB wet etching, several settings have been investigated to produce and achieve better structure of circular FSS. The following settings is the best settings as compared to the others setting of wet etching process as it produces a better structure of circular FSS as shown in Fig. 3, even though there is a slight transformation to a diamond like shape. As a further step to the fabrication process, the pattern on masking film is exposed under UV light to transfer the pattern from the masking film to roger surface. Later, the pattern on the Roger board has been immersed into developing solution of sodium hydroxide to remove the unwanted photoresist layer. Several parameters such as time and temperature of the machine have been varied to obtain the best structure of FSS during developing process. The best structure that can be obtained during the fabrication is at a temperature of $30^{\circ} \mathrm{C}$ with time taken to immersed in the solution is about 30 second. Furthermore, during etching process, all the unwanted copper has been removed using ferric chloride acid for about 2 minutes with constant temperature of $43-44^{\circ} \mathrm{C}$. Lastly, the FSS structure has been put into the stripping machine for rinsing for approximately 1 minute under a constant temperature of $44^{\circ} \mathrm{C}$ before dry it out. 


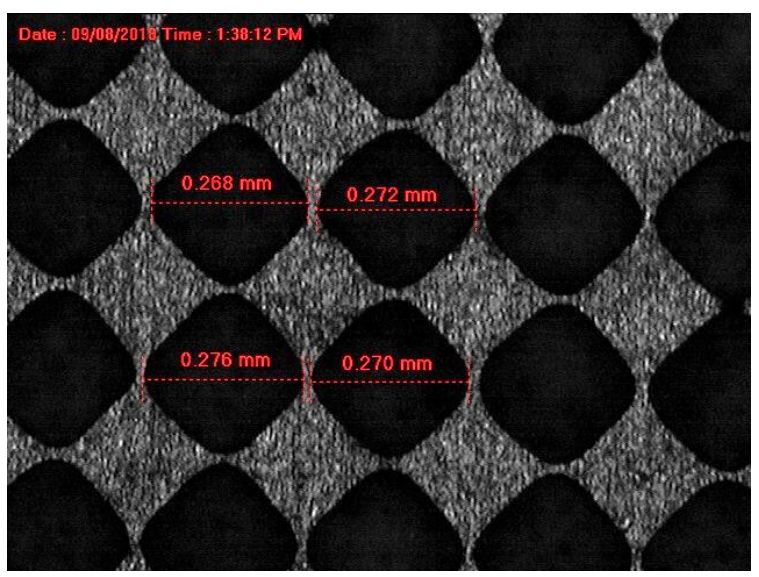

Fig. 3 - Structure of circular slot FSS under microscope

\section{Result and discussion}

The FSSs are etched as $41 \times 41$ elements on Rogers RT5880LZ board with size of more than 10 wavelengths at each side. Unit cell setup during simulation can represent as infinite array that consist of identical elements by a plane wave in the solver during the simulation [17]. Moreover, in this paper, circular slot structure is chosen because of its simple and basic structure in order to encounter the limitation of wet etching technique. The fabricated FSS later has been measured using terahertz time domain spectroscopy for measured transmission result.

Circular slot FSS has been designed to operate at frequency $0.66 \mathrm{THz}$. From the measured results show good agreement, as shown in Fig 4, even though there is slightly alteration into diamond shape structure after fabrication process due to the limitation of wet etching as previously shown in Fig 3 .

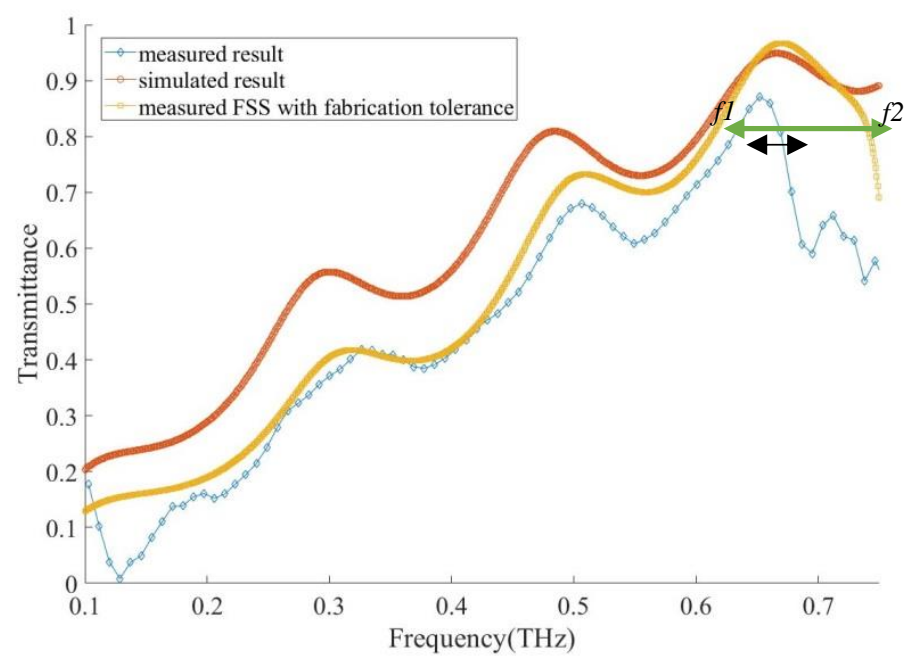

Fig. 4 - Comparison between simulated, measured result and measured with fabrication tolerance of circular FSS

Result on figure 4 shows $1 \%$ frequency shifts from $0.66 \mathrm{THz}$ to $0.65 \mathrm{THz}$ with a magnitude transmission difference of 0.0776. Moreover, fabrication of circular FSS shows narrower bandwidth as compared to the simulated result. Similar fabrication technique from other research paper also obtain similar amount of differences with different geometries of FSS [13].

Furthermore, the inspection through Stereo Zoom Microscope for the comparison between structures of before and after fabrication process is investigated. Observation shows that, after the fabrication, the diameter structure of the FSS has deviated inconsistency from $0.268 \mathrm{~mm}$ to $0.276 \mathrm{~mm}$ by actual design modelled in CST Microwave. The fabricated structure of FSS approximately becomes $3 \%$ to $6 \%$ smaller as compared to the actual structures after fabrication. Another Inspection has been observed is the thickness of metal layer copper. Field-Emission Scanning Electron Microscope (FESEM) is used to observe the thickness of the copper layer. From the FESEM result shows that, the layer of copper thickness on top of the FSS becomes $0.0133 \mathrm{~mm}(13.30 \mu \mathrm{m})$ thinner from actual copper thickness in simulated FSS that 
is $0.035 \mathrm{~mm}(35 \mu \mathrm{m})$. The results of fabrication tolerance such as, deviation of copper thickness, and alteration of circular structure have been adapted into CST simulation to observe the fabrication effect on the FSS transmission. Hence, as shown in Fig.4, the simulated structure of FSS after it is adapted by fabrication tolerance shows closely resemblance to simulated results of circular structure, if the diameter of diamond shape is nearly same as circular structure. However, with the fabrication tolerance, it is shows that FSS surface current intensity remain unchanged hence, showed that FSS structure able to increase the detection sensitivity in material sensing application.

Despite the limitations of wet etching, basic and simple structure of Circular FSS is able to be realized and obtained good transmission magnitude value of approximately 0.871 as compared to the studies mentioned in [13] and [18]. The cited works have also used wet etching process to fabricate complicated structures like Jerusalem-cross, cross-shaped and U-shaped. Thus, the mentioned designs produce lower transmittance magnitude than circular slot FSS as shown in Table 1.

Table 1 - Comparison study on the fabrication of FSS

\begin{tabular}{cccc}
\hline \multirow{2}{*}{ Ref } & $\begin{array}{c}\text { Fabrication } \\
\text { Technique }\end{array}$ & $\begin{array}{c}\text { Geometries } \\
\text { involved }\end{array}$ & $\begin{array}{c}\text { Result } \\
\text { (transmittance } \\
\text { magnitude) }\end{array}$ \\
\hline$[13]$ & Wet Etching & $\begin{array}{c}\text { U-shaped, } \\
\text { Jerusalem } \\
\text { Cross } \\
\text { shaped, } \\
\text { Cross- } \\
\text { shaped } \\
\text { Cross- } \\
\text { dipole } \\
\text { shaped } \\
\text { Circular } \\
\text { Shaped }\end{array}$ & 0.400 \\
T18] & Wet etching & 0.646 \\
This paper & & 0.871 \\
\hline
\end{tabular}

\section{Conclusion}

This paper proves that the realisation of the circular slot FSS is possible with photoplotter and wet etching technique. With limitation of wet etching technique, only small shifts are identified by comparison with simulated and measured result. Besides that, measured result of circular FSS able obtain the transmission magnitude of 0.871. Overall, the results show that a good agreement is obtained between simulated and measured results, with able to achieve $87 \%$ of its transmittance peak using basic and simple structure such circular. In the future, the fabricated FSS will be measured with the specified nonconductive materials to demonstrate its capability to increase the accuracy of detection in the $\mathrm{THz}$ spectroscopy material sensing.

\section{Acknowledgement}

The authors wish to acknowledge the funding of this project by Fundamental Research Grant (FRGS vot: 1629) and GPPS Grant VOT H026, Ministry of Education Malaysia, Registrar Office and Research Management Centre (RMC) of Universiti Tun Hussein Onn Malaysia (UTHM).

\section{References}

[1] P. Garbacz. (2016). Terahertz imaging - principles, techniques, and limitations, Maint. Probl., 81-92

[2] J. Qin, L. Xie, and Y. Ying. (2016). A high-sensitivity terahertz spectroscopy technology for tetracycline hydrochloride detection using metamaterials, Food Chem., 211, 300-305

[3] C. Song, W. H. Fan, L. Ding, X. Chen, Z. Y. Chen, and K. Wang. (2018). Terahertz and infrared characteristic absorption spectra of aqueous glucose and fructose solutions, Sci. Rep., 8, 2-9

[4] W. T. Liu, J. W. Li, and W. Yang. (2010). Detection and identification of explosives and illicit drugs by terahertz spectroscopy technology, Int. Geosci. Remote Sens. Symp., 3576-3579

[5] U. Moller, H. Merbold, J. R. Folkenberg, and P. U. Jepsen. (2007). Determination of alcohol- and sugar concentration in aqueous solutions using reflection terahertz time-domain spectroscopy, 2007 Jt. 32nd Int. Conf. Infrared Millim. Waves 15th Int. Conf. Terahertz Electron., 1-2

[6] B. Qin et al. (2018). Highly Sensitive Detection of Carbendazim by Using Terahertz Time-Domain Spectroscopy Combined with Metamaterial, IEEE Trans. Terahertz Sci. Technol., 8, 149-154

[7] D. K. Lee et al. (2016). Ultrasensitive Detection of Residual Pesticides Using THz Near-Field Enhancement, 
IEEE Trans. Terahertz Sci. Technol., 6, 389-395

[8] K. Wang, D. W. Sun, and H. Pu. (2017). Emerging non-destructive terahertz spectroscopic imaging technique: Principle and applications in the agri-food industry, Trends in Food Science and Technology, 67, 93-105

[9] S. S. Yadav, A. Sonker, and A. K. Palange. (2016). Parametric study on frequency selective surfaces, 2016 International Conference on Communication and Signal Processing (ICCSP), 499-502.

[10] B. A. Munk (2000). Frequency Selective Surfaces: Theory and Design. Wiley-Interscience Pub.

[11] J. Li, Y. Li, and L. Zhang. (2017). Terahertz Bandpass Filter Based on Frequency Selective Surface, IEEE Photonics Technol. Lett., 30, 1.

[12] X. Chen, Y. Chen, M. Yan, M. Qiu, and T. Cui. (2012). Photothermal direct writing of metallic microstructure for frequency selective surface at terahertz frequencies, Proc. 2012 Int. Work. Metamaterials, Meta 2012, 1-3.

[13] Y. Zhu, S. Vegesna, V. Kuryatkov, M. Holtz, M. Saed, and A. Bernussi. (2011). THz time-domain spectroscopy of multilayer filters, IRMMW-THz 2011 - 36th International Conference on Infrared, Millimeter, and Terahertz Waves, 161-162

[14] F. C. Seman and N. K. Khalid. (2015). Design strategy for optimum planar square loop FSS with different dielectric substrates, Lecture Notes in Electrical Engineering

[15] D. K. Lee et al. (2016). Ultrasensitive Detection of Residual Pesticides Using THz Near-Field Enhancement, IEEE Trans. Terahertz Sci. Technol., 6, 389-395

[16] S. Niknam, M. Yazdi, and S. B. Amlashi. (2019). Enhanced Ultra-Sensitive Metamaterial Resonance Sensor based on Double Corrugated Metal stripe for Terahertz Sensing, Sci. Rep., 2-10

[17] M. Zhou et al. (2017). Design of aperiodic frequency selective surfaces for compact quasi-optical networks, 11th Eur. Conf. Antennas Propagation, EUCAP 2017, 1274-1278

[18] D. S. Wang, B. J. Chen, and C. H. Cha. (2016). High-Selectivity Bandpass Frequency-Selective Surface in Terahertz Band, IEEE Trans. Terahertz Sci. Technol., 6, 284-291 